Letter to the Editor

\title{
Vitamin D epimer: a significant bioregulator or an inactive compound?
}

Spyridon Karras (1), Andrea Petroczi (2), Panagiotis Anagnostis (1), Naughton P. Declan (2)

(1) Unit of Reproductive Endocrinology, First Department of Obstetrics and Gynecology, Medical School, Aristotle University of Thessaloniki, Thessaloniki, Greece

(2) School of Life Sciences, Kingston University, Kingston Upon Thames, London, United Kingdom

Corresponding author and author to whom requests for reprints should be addressed:

Spyridon N Karras, Venizelou 34b,Pilea ,Thessaloniki,Greece, Tel:+00302373021922

Fax: +00302373021922

karraspiros@yahoo.gr

Spyridon Karras, Andrea Petroczi, Panagiotis Anagnostis and Declan P. Naughton declare that they have no conflict of interest. 


\section{Dear Editor,}

We read with interest the study by Chailurkit et al [1], published recently in your journal. The authors provide a global report regarding the percentage of $\mathrm{C} 3$ epimer of $25(\mathrm{OH}) \mathrm{D}$ and its determinants in a representative sample of an Asian adult population. In concordance with previous observations, serum 3-epi-25(OH) D3 was detected in all samples, ranging from 1.8 to $24.8 \%$ of total $25(\mathrm{OH}) \mathrm{D} 3$, with the majority of distribution accounting for approximately $5-10 \%$. Interesting correlations regarding age, male gender and rural residence were also evident, although they predicted only a small amount of variation of 3-epi-25(OH) D3 /total 25(OH) D3 ratio.

Particular considerations might be of interest towards a more holistic interpretation of these findings. First of all, as the scientific community elapses the era of vitamin D supplementation for disease outcomes, we consider that until the potential biological actions of 3-epi-25(OH) D3, are elucidated from future studies, assaying the epimers separately could provide the most rational way of understanding, targeting and treating vitamin D deficiency and insufficiency. In clinical terms, if epimers represent a biologically inactive compound of vitamin homeodynamics, the rough estimates of $25(\mathrm{OH}) \mathrm{D}$ reserve, although not altered in this study, are likely to be affected, particularly in supplementation trials where vitamin D3 is used. The study by Chailurkit et al [1] provides data from samples with no previous vitamin D3 supplementation. On the other hand, if future studies derive a biological role for 3- 
epi-25(OH) D3, it could be hypothesized that it manifests a distinct role to that of $25(\mathrm{OH}) \mathrm{D} 3$.

Secondly, previous data have reported that epimers might play a role in disease activity in several diseases, including Alzheimer's disease, type 1 diabetes and rheumatoid arthritis [2]. Analysis of 10 forms of vitamin D revealed that the 3-epi$25(\mathrm{OH}) \mathrm{D} 3$ form is present at significantly higher levels in those suffering from $\mathrm{s}$ Alzheimer disease, type 1 diabetes and rheumatoid arthritis [healthy controls $(6.093 \pm$ $6.711 \mathrm{nmol} / \mathrm{L} ;$ combined disease $=22.433 \pm 13.535 \mathrm{nmol} / \mathrm{L}, t(52.5)=-6.411 ; P<$ $.001]$. Furthermore, both 3-epi-25(OH)D level and proportion, but not the epimerfree 25OHD, showed good diagnostic power for the chronic disease status (AUC $=$ $0.880, P<.001)$. These results warrant further investigation in large scale studies, along with an expansion to other chronic diseases that have been previously linked to vitamin D.

Finally, we would like to emphasize on the need for similar studies from a wide range of infant samples. While, previous data indicated that neonatal and infant vitamin D stores are reliant from maternal ones [3], routine accurate measurement of epimers, could overcome the technical flaws provoked by the presence of a significant percentage of epimers as a fraction of total vitamin D levels. 


\section{References}

1. Chailurkit L, Aekplakorn W, Ongphiphadhanakul B. Serum C3 epimer of 25hydroxyvitamin D and its determinants in adults: a national health examination survey in Thais. Osteoporos Int. 2015 Apr 14. [Epub ahead of print]

2.Shah I, Petroczi A, Naughton DP (2014) Exploring the roles of vitamin D in Type 1 diabetes, rheumatoid arthritis, and Alzheimer Disease: New insights from accurate analysis of 10 forms. J Clin Endocrinol Metab 99:808-816.

3. Karras SN, Shah I, Petroczi A, Goulis DG, Bili H, Papadopoulou F, Harizopoulou V, Tarlatzis BC, Naughton DP. An observational study reveals that neonatal vitamin $\mathrm{D}$ is primarily determined by maternal contributions: implications of a new assay on the roles of vitamin D forms. Nutr J. 2013 Jun 7;12:77. 